\title{
Degradation of low rank coal by Trichoderma atroviride ES11
}

\author{
M. Estela Silva-Stenico • Caryn J. Vengadajellum • \\ Hussnain A. Janjua · Sue T. L. Harrison • \\ Stephanie G. Burton · Don A. Cowan
}

Published online: 25 July 2007

(C) Society for Industrial Microbiology 2007

\section{Erratum to: J Ind Microbiol Biotechnol \\ DOI 10.1007/s10295-007-0223-7}

Unfortunately, a wrong version of Fig. 2 was published in the article. The correct figure is given below:
The online version of the original article can be found under doi:10.1007/s10295-007-0223-7.

M. E. Silva-Stenico $\cdot$ H. A. Janjua $\cdot$ D. A. Cowan $(\varangle)$ Advanced Research Centre for Applied Microbiology, Department of Biotechnology, University of the Western Cape, Private Bag X17, Bellville, 7535 Cape Town, South Africa e-mail: dcowan@uwc.ac.za

\section{J. Vengadajellum · S. T. L. Harrison · S. G. Burton} Bioprocess Engineering Research Unit, Department of Chemical Engineering, University of Cape Town, Rondebosch, 7700 Cape Town, South Africa
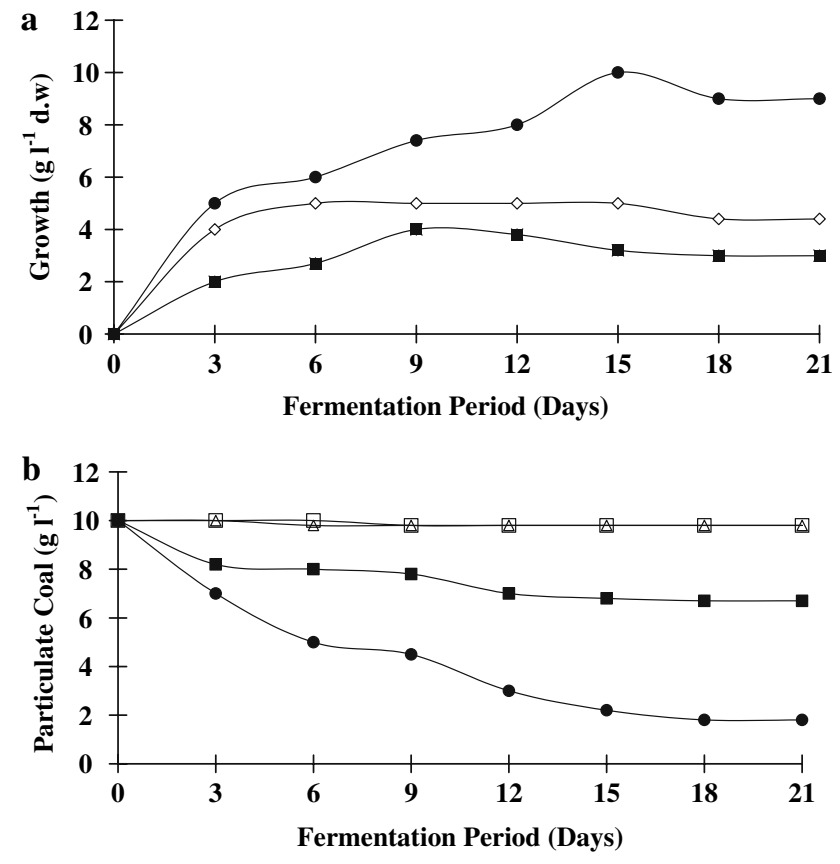

Fig. 2 Biomass yield and coal degradation for T. atroviride ES11 shake flask experiments. Fungal mycelia were grown at $28^{\circ} \mathrm{C}$ in $3 \%$ malt extract medium until exponential growth was achieved. This culture was used to inoculate malt extract medium containing coal $\left(10 \mathrm{~g}^{-1}\right.$; filled box) or coal plus glucose $\left(5 \mathrm{~g}^{-1}\right.$; filled circle $)$. In the biomass yield control flask fungal mycelia were grown in medium supplemented with glucose (open diamond). Controls for coal degradation analysis consisted of growth medium containing coal (open triangle) or coal plus glucose (open box), incubated without mycelium inoculation. Biomass yield (a) and coal degradation (b) were measured over a period of 21 days. Samples were taken after every 3 days 